\title{
Delayed variations in the diagnostic accuracy of intraoperative neuromonitoring in the resection of intramedullary spinal cord tumors
}

\author{
Gil Kimchi, MD, ${ }^{1}$ Nachshon Knoller, MD, ${ }^{1}$ Akiva Korn, MMedSc, D-ABNM, ${ }^{2}$ Yahel Eyal-Mazuz, MSc, ${ }^{2}$ \\ Yechiam Sapir, PhD, ${ }^{2}$ Anton Peled, MD, ${ }^{1}$ and Ran Harel, MD'
}

1'Department of Neurosurgery, Sheba Medical Center, Ramat-Gan, Israel, affiliated with Sackler School of Medicine, Tel Aviv University, Tel Aviv; and 2Surgical Monitoring Services Ltd., Beit Shemesh, Israel

\begin{abstract}
OBJECTIVE The use of intraoperative neuromonitoring (IONM) has become an imperative adjunct to the resection of intramedullary spinal cord tumors (IMSCTs). While the diagnostic utility of IONM during the immediate postoperative period has been previously studied, its long-term diagnostic accuracy has seldom been thoroughly assessed. The aim of this study was to evaluate long-term variations in the diagnostic accuracy of transcranial motor evoked potentials (tcMEPs), somatosensory evoked potentials (SSEPs), and D-wave recordings during IMSCT excision.
\end{abstract}

METHODS The authors performed a retrospective evaluation of imaging studies, patient charts, operative reports, and IONM recordings of patients who were operated on for gross-total or subtotal resection of IMSCTs at a single institution between 2012 and 2018. Variations in the specificity, sensitivity, positive predictive value (PPV), and negative predictive value (NPV) for postoperative functional outcome (McCormick Scale) were analyzed at postoperative day 1 (POD1), 6 weeks postoperatively (PO-6 weeks), and at the latest follow-up.

RESULTS Overall, 28 patients were included. The mean length of follow-up was $19 \pm 23.4$ months. Persistent motor attenuations occurred in $71.4 \%$ of the cohort. MEP was the most sensitive modality $(78.6 \%, 87.5 \%$, and $85.7 \%$ sensitivity at POD1, PO-6 weeks, and last follow-up, respectively). The specificity of the D-wave was the most consistent over time $(100 \%, 83.35 \%$, and $90 \%$ specificity at the aforementioned time points). The PPV of motor recordings decreased over time (58\% vs $33 \%$ and $100 \%$ vs 0 for tcMEP and D-wave at POD1 and last follow-up, respectively), while their NPV consistently increased $(67 \%$ vs $89 \%$ and $70 \%$ vs $100 \%$ for tcMEP and D-wave at POD1 and last follow-up, respectively).

CONCLUSIONS The diagnostic accuracy of IONM in the resection of IMSCTs varies during the postoperative period. The decrease in the PPV of motor recordings over time suggests that this method is more predictive of short-term rather than long-term neurological deficits. The increasing NPV of motor recordings indicates a higher diagnostic accuracy in the identification of patients who preserve neurological function, albeit with an increased proportion of false-negative alarms for the immediate postoperative period. These variations should be considered in the surgical decision-making process when weighing the risk of resection-associated neurological injury against the implications of incomplete tumor resection. https://thejns.org/doi/abs/10.3171/2021.2.FOCUS201084

KEYWORDS intramedullary tumors; intraoperative neuromonitoring; spine oncology

I NTRAMEDULLARY spinal cord tumors (IMSCTs) are uncommon pathological entities that may lead to significant neurological deficits. The limited role of nonoperative treatment renders these pathologies amenable for surgical intervention in the majority of cases. ${ }^{1,2}$ Despite advancements in neurosurgical techniques and assistive devices, IMSCT resection still carries a considerable risk for neurological deterioration, reaching as high as $90 \%$ in the immediate postoperative period. ${ }^{3}$ With benign pathologies such as ependymoma being the most common in the adult population, ${ }^{2,4,5}$ gross-total resection (GTR) is pursued in the majority of cases. ${ }^{1,6}$ In more infiltrative pathologies, i.e., astrocytoma, a subtotal resection (STR) may be adequate to reduce resection-associated neurological injury. To mitigate the exceedingly high neurological deterioration rates associated with the excision of intramedullary tumors, the use of intraoperative neuromonitoring (IONM) has become an imperative adjunct to their resection. Motor

ABBREVIATIONS GTR = gross-total resection; IMSCT = intramedullary spinal cord tumor; IONM = intraoperative neuromonitoring; IQR = interquartile range; MEP = motor evoked potential; NPV = negative predictive value; POD = postoperative day; PO- 6 weeks = 6 weeks postoperatively; $\mathrm{PPV}=$ positive predictive value; PTN = posterior tibial nerve; SSEP = somatosensory evoked potential; STR = subtotal resection; tcMEP = transcranial MEP; ULN = ulnar nerve.

SUBMITTED December 23, 2020. ACCEPTED February 22, 2021.

INCLUDE WHEN CITING DOI: 10.3171/2021.2.FOCUS201084. 
evoked potentials (MEPs), somatosensory evoked potentials (SSEPs), electromyography, and D-wave recordings are the modalities most commonly used., ${ }^{1,7}$ While the diagnostic utility of IONM during the immediate postoperative period has been previously studied, its long-term diagnostic accuracy has seldom been thoroughly assessed.7-10 The aim of this study was to describe temporal variations in the diagnostic accuracy of MEP, SSEP, and D-wave recordings at various time points following the excision of IMSCTs and investigate their implications for the surgical decision-making process.

\section{Methods}

After institutional review board approval was obtained for this study, we performed a retrospective evaluation of imaging studies, patient charts, operative reports, and IONM recordings (MEP, SSEP, and D-wave) of patients who were operated on for GTR or STR of IMSCTs in a single institution between the years 2011 and 2018. All adult ( $\geq 18$ years old) patients who were diagnosed by MRI with an IMSCT from various pathologies at any spinal level were included in the study. Exclusion criteria included 1) repeated tumor resection, 2) surgery limited to spinal cord biopsies, and 3) IONM recordings not obtained ( $\mathrm{n}=1$, due to an intratumoral hemorrhage with rapid neurological deterioration that required an expedient surgical intervention). The primary outcome was postoperative functional status, classified by using the McCormick Scale. ${ }^{3}$ In addition to the preoperative evaluation, functional assessment was performed at postoperative day 1 (POD1), 6 weeks postoperatively (PO-6 weeks), and the latest follow-up.

\section{Statistical Analysis}

The specificity, sensitivity, positive predictive value (PPV), and negative predictive value (NPV) of transcranial MEP (tcMEP), SSEP, and D-wave recordings were evaluated for changes in the postoperative McCormick Scale grade. ${ }^{3}$ Continuous variables were evaluated for normal distribution using a histogram and described as median and interquartile range (IQR). Categorical variables were described as frequency and percentage. Nonparametric tests were used due to the small sample size. Fisher's exact test was used to evaluate the association between IONM attenuation and postoperative outcome. Values were considered statistically significant at $\mathrm{p}<0.05$. All statistical analyses were two-sided. IBM SPSS software (version 25; IBM Corp.) was used for all statistical analyses, and Excel software (Microsoft Corp.) was used to create profile plots.

\section{Surgical Technique}

All patients were placed in the prone position, with the head secured in a 3-pin head holder for lesions above the T6 vertebra. Total intravenous anesthesia was administered in all cases. Midline myelotomy was employed in all but two cases, in which a dorsal root entry zone incision was performed. Tumor resection was carried out in a combined technique of blunt and sharp dissection, with the occasional introduction of an ultrasonic aspirator (CUSA, Integra LifeSciences Corp.). Instrumentation was not implanted in any of the cases. In the presence of motor or somatosensory attenuations, the following workflow steps were employed: 1) assuring adequate blood pressure, 2) evaluating depth of anesthesia using EEG recording and ruling out oversedation, and 3) assuring normothermia.

\section{Electrophysiological Monitoring}

IONM consisted of recordings of tcMEP, D-wave, and muscle generators, SSEP, and free-running electromyography using the NIM-Eclipse (Medtronic Corp.) recording system.

\section{Somatosensory Evoked Potential}

Upper-extremity sensory activity was recorded by placing surface electrodes along the lateral aspect of each wrist for bipolar stimulation of the ulnar nerves (ULNs). ULN stimulations were made using a pulse duration of $200 \mu \mathrm{sec}$, intensity of $25-45 \mathrm{~mA}$, and repetition rate of 2.3-4.1 Hz. Data were recorded over the cerebral cortex using subdermal corkscrew electrodes at standard scalp positions, according to the International 10-20 electroencephalography system. Lower-extremity activity was recorded by placing surface electrodes along the medial aspect of each ankle at the malleoli for bipolar stimulation of the posterior tibial nerves (PTNs). Recordings were made using 200- $\mu$ sec pulse duration, 35- to 45-mA intensity, and 2.3- to $4.1-\mathrm{Hz}$ repetition rate. Averages of 50-300 sweeps were collected in accordance with the signal-tonoise ratio. Baseline data were taken prior to skin incision, and amplitude of the sensory responses was set according to the cortical generator potential peak (N20 for ULN and P37 from PTN) compared with the onset. The latency and amplitude of these potentials were sampled every 5-15 minutes, allowing the identification and monitoring of impairments of the somatosensory pathway. Warning criteria for SSEPs were defined as a 50\% decrease in amplitude and/or a $10 \%$ increase in latency of the signal compared with baseline values.

\section{Transcranial Electric MEPs}

Subdermal corkscrew electrodes were placed approximately $1 \mathrm{~cm}$ anterior and $2 \mathrm{~cm}$ medial to $\mathrm{C} 3$ and $\mathrm{C} 4$ scalp positions according to the International 10-20 system for transcranial stimulation. For cervical lesions, data were recorded from bilateral deltoid, biceps, triceps, thenar, and abductor hallucis muscles, and for thoracic lesions, data were recorded from thenar, quadriceps, anterior tibialis, and abductor hallucis muscles. A ground electrode was placed at one of the shoulders. Single train stimulation (7 biphasic pulses or 9 normal/inverse pulses) was delivered at $250-333 \mathrm{~Hz}$, with an intensity range of $60-400 \mathrm{~V}$ and $500-\mu \mathrm{sec}$ pulse duration. The frequency of stimulation and tcMEP data assessment varied according to the sensitivity of the surgical stage. During critical stages, data were sampled every 5-10 minutes, while during noncritical parts of surgery, or when use of electrocautery was necessary, as during exposure, stimulation was undertaken less frequently. Warning criteria were established as a persistent attenuation of more than $80 \%$ in the motor response amplitude for segmental MEPs and/or abolishment of the long-tract MEPs. 
TABLE 1. Patient characteristics

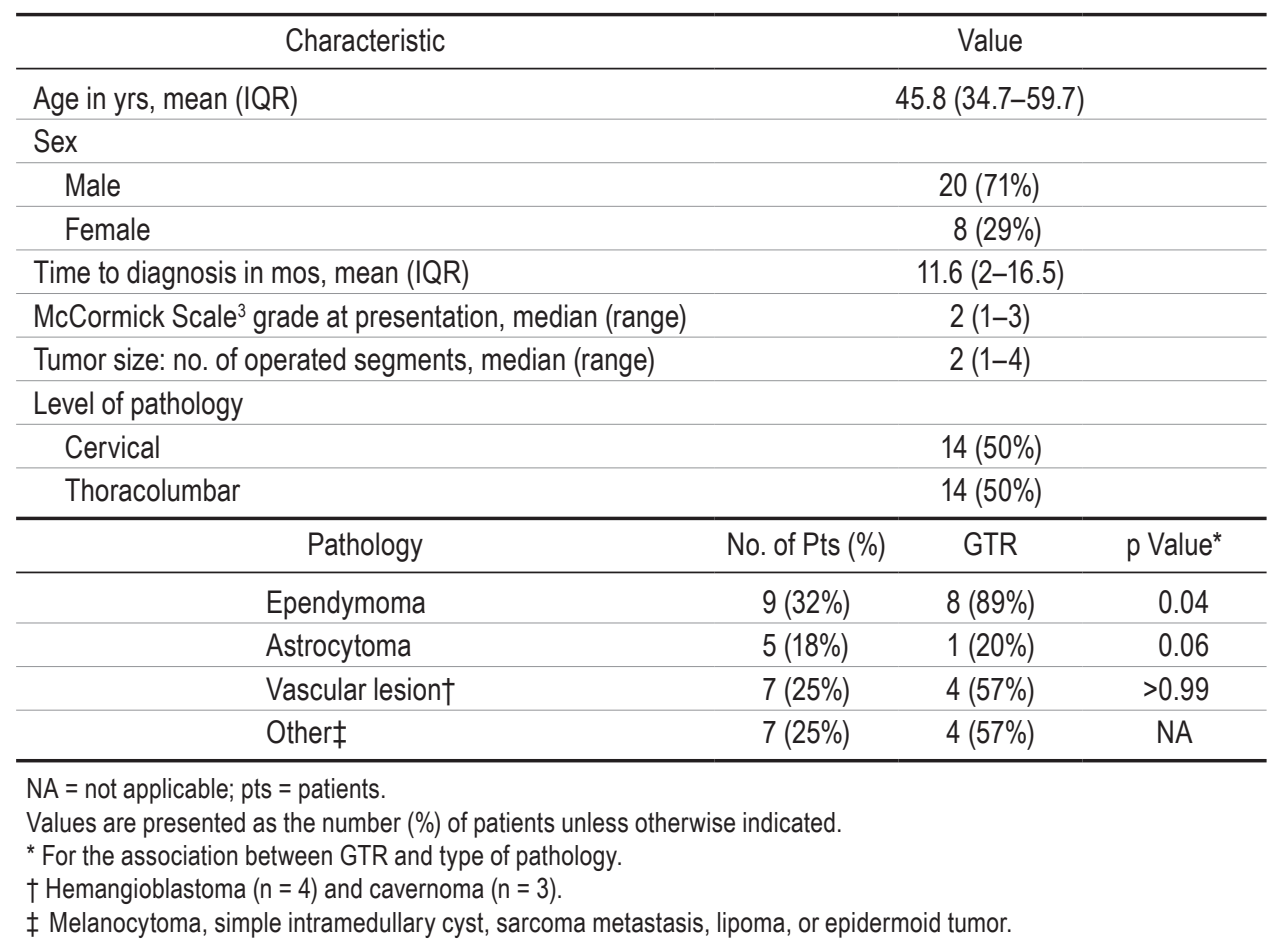

\section{Transcranial Electric Spinal Direct Waves (D-waves)}

Two- or three-contact catheter-type electrodes (AdTech) were inserted into the subdural space at the levels above (rostral [control]) and below (caudal) the tumor in order to record descending spinal cord motor pathways. D-waves were induced by the same cephalic stimulating electrodes used for tcMEPs except with single pulses $(500$ $\mu \mathrm{sec})$. An average of 5-25 50-msec sweeps were utilized when necessary. Baseline was established prior to tumor resection; data were recorded continuously during resection and were compared to baseline. D-wave recordings were not attempted when the tumor level was below T10 or when dural adhesions disturbed electrode placement. In high cervical tumors, the rostral electrode was not inserted. An absolute warning criterion for D-waves was defined as $a \geq 50 \%$ decrease in amplitude of the caudal potential compared with baseline.

\section{Results}

Overall, 28 patients met the inclusion criteria for this study. Patient characteristics are displayed in Table 1. Follow-up examinations were completed in $100 \%$ and $78.5 \%$ of cases at POD1 and PO-6 weeks, respectively. The mean length of follow-up was $19 \pm 23.4$ months. GTR was achieved in 17 operations (60.7\%) and STR was performed in 11 operations (39.3\%). The association between GTR and pathology type is displayed in Table 1.

\section{IONM Attenuations}

SSEPs and MEPs were recorded in all cases. D-wave recordings were obtained in 12 cases $(42.8 \%)$. Attenuation of potentials was identified in $82.1 \%$ of the cohort ( $\mathrm{n}$
= 23) in the following modalities: SSEP, 7 cases (25\%); MEP, 20 cases (71.4\%); and D-wave, 2 cases (16.6\%). In 3 operations (13\%), the potentials recovered to their baseline level by the end of surgery. In all of these cases, the surgeon opted to continue the resection despite the neuromonitoring alarm. Tumor excision was halted in 7 operations $(24 \%)$ due to persistent motor attenuations (tcMEP or D-wave). Intravenous steroids were administered once attenuations were recorded.

\section{Diagnostic Accuracy of IONM}

Diagnostic statistics of IONM are displayed in Table 2. Attenuations in tcMEPs were not significantly associated with postoperative deterioration at any of the postoperative time points $(p>0.99, p=0.21$, and $p>0.99$ at POD1, PO-6 weeks, and latest follow-up, respectively), as well as attenuations in SSEPs $(\mathrm{p}=0.66, \mathrm{p}=0.31$, and $\mathrm{p}=0.06$ at POD1, PO-6 weeks, and last follow-up, respectively). The number of operated levels, extent of resection, patient age, pathology type, and D-wave attenuations were not significantly associated with postoperative deterioration. Postoperative functional status is summarized in Table 3. Profile plots for the change in McCormick Scale grade over time are displayed in Fig. 1.

The mean postoperative length of stay was 9 days (IQR 4-13 days). Twelve patients (42.8\%) were discharged to their homes and the remainder (67.2\%) to a rehabilitation facility. One patient had a superficial wound infection that was treated with intravenous antibiotics. One patient had a CSF leak without meningitis; he was treated conservatively with the insertion of a continuous intrathecal drain for 5 days, thereupon the leak had ceased. 
TABLE 2. Diagnostic statistics of IONM

\begin{tabular}{lccc}
\hline & SSEP (\%) & tcMEP (\%) & D-Wave (\%) \\
\hline Sensitivity & & & \\
\hline POD1 & 21.4 & 78.6 & 40 \\
\hline PO-6 wks & 25 & 87.5 & 33.3 \\
\hline Last follow-up & 0 & 85.7 & 100 \\
\hline Specificity & & & \\
\hline POD1 & 71.4 & 42.9 & 100 \\
\hline PO-6 wks & 78.6 & 50 & 83.3 \\
\hline Last follow-up & 70 & 40 & 90 \\
\hline NPV & & & \\
\hline POD1 & 47.6 & 66.7 & 70 \\
\hline PO-6 wks & 64.7 & 87.5 & 71.4 \\
\hline Last follow-up & 66.7 & 88.9 & 100 \\
\hline PPV & & & \\
\hline POD1 & 42.9 & 57.9 & 100 \\
\hline PO-6 wks & 40 & 50 & 50 \\
\hline Last follow-up & 0 & 33.3 & 50 \\
\hline
\end{tabular}

\section{Illustrative Case}

An otherwise healthy 37-year-old male patient was referred to our outpatient Neurosurgical Spine Clinic due to progressive paraparesis and bilateral lower-extremity dysesthesias for the previous 6 months. He reported a gradually worsening gait instability, increased urinary frequency, and sexual dysfunction. His neurological examination was significant for a T2 sensory level, proximal paraparesis, and patellar hyperreflexia. His functional status was classified as McCormick grade II. His cervicothoracic MRI (Fig. 2A and B) was notable for a contrast-enhancing intramedullary expansile lesion with a cystic component at the C7-T3 levels, compatible with intramedullary ependymoma. Complete neuroaxis MRI ruled out additional lesions. Ten days following his diagnosis, a microscopyassisted excision of the tumor was performed. MEP, SSEP, and D-wave recordings were obtained. At baseline, MEPs and SSEPs were normal in all extremities, but the D-wave recordings were slightly attenuated yet stable. Following a midline posterior myelotomy, a vascularized mass with surrounding intramedullary edema was observed. A frozen section confirmed the diagnosis of ependymoma. As the resection advanced to the margins of the lesion, abolishment of the iliopsoas MEPs was recorded bilaterally with albeit stable D-wave and SSEP recordings. The declining potentials prompted the administration of intravenous steroids and the increase in mean arterial blood pressure, yet no recovery of potentials was observed. As residual tumor mass was still visible and ongoing bleeding was evident, the surgeon decided to carry on the excision despite the discrepant potential recordings until GTR was achieved. The MEP and D-wave recordings remained unchanged during the procedure. Immediately following the operation, the patient developed significant paraparesis involving proximal and distal muscle groups, accompanied by worsening proprioception and light touch impairment. His status was classified as McCormick grade IV. After an otherwise uneventful postoperative course, initial sensory improvement was observed on POD5. At PO-6 weeks the patient began ambulating with the assistance of external aids, and by 6 months postoperatively he was ambulating independently. At the time of this report his latest followup MRI had been performed 21 months following surgery and demonstrated GTR of the lesion without recurrence (Fig. 2C and D). The definitive pathological diagnosis was grade II ependymoma.

\section{Discussion}

The use of IONM is considered a standard of care in the resection of IMSCT. Ideally, it alarms the surgeon before permanent neurological injury occurs and improves patient safety and postoperative outcomes. Notwithstanding, attenuations of recordings may occur in the midst of tumor excision and the surgical team can be faced with a dilemma when weighing the risk of resection-associated neurological injury against the implications of STR.

In this study, we analyzed changes in the diagnostic accuracy of IONM related to the prediction of postoperative outcomes during three time points and found significant variations in the sensitivity, albeit a relatively consistent specificity for all neuromonitoring modalities. In this cohort, the PPV of motor recordings for postoperative neurological deterioration has decreased by $25 \%$ over time (58\% vs $33 \%$ and $100 \%$ vs 0 for tcMEP and D-wave at POD1 and last follow-up, respectively), suggesting it is more predictive of short-term rather than long-term neurological deficits. In addition, the NPV has consistently increased (67\% vs $89 \%$ and $70 \%$ vs $100 \%$ for tcMEP and D-wave at POD1 and last follow-up, respectively), indicating a higher diagnostic accuracy in the identification of patients who eventually preserve neurological function, albeit an increased proportion of false-negative results in the short-term perioperative period. Of note, in contrast to tcMEP and SSEP monitoring, the limited role of D-

TABLE 3. Postoperative outcome

\begin{tabular}{cclcc}
\hline McCormick Scale Grade & Baseline & POD1 & PO-6 Wks & Last Follow-Up \\
\hline I & $11(39)$ & $7(25)$ & $8(36.4)$ & $13(43.4)$ \\
II & $10(35.7)$ & $5(17.8)$ & $4(18.2)$ & $1(3.5)$ \\
\hline III & $7(25)$ & $7(25)$ & $7(31.8)$ & $8(28.5)$ \\
\hline IV & 0 & $9(32.1)$ & $3(13.6)$ & $4(14.3)$ \\
\hline
\end{tabular}

Values are presented as the number (\%) of patients. 

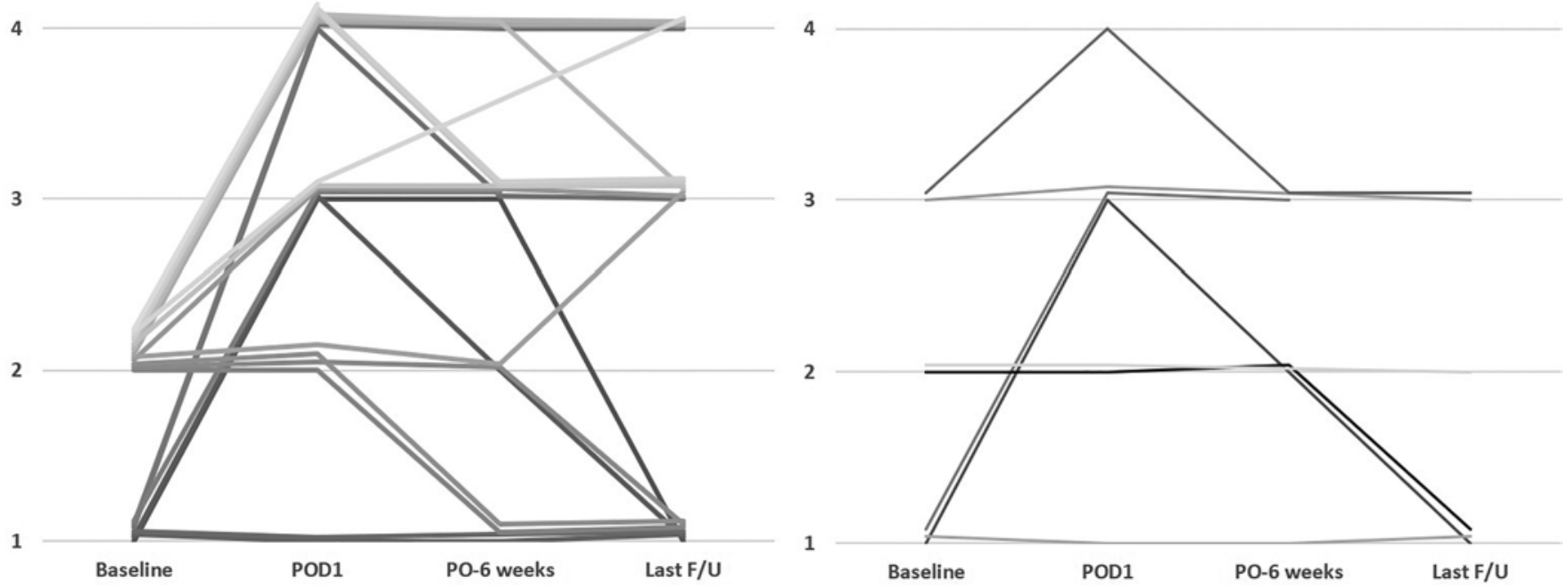

FIG. 1. McCormick Scale score for patients with IONM attenuations: tcMEP attenuations $(\mathbf{A})$ and SSEP attenuations $(\mathbf{B})$. F/U = follow-up; Pts = patients.

wave recordings to herald early postoperative outcomes was demonstrated in multiple publications that alluded this modality is more predictive of long-term rather than short-term outcomes. ${ }^{8,11-13}$ In our cohort, the sensitivity and NPV of D-wave recordings increased from POD1 onward, emphasizing the importance of correlating the IONM modality with the time point in which its predictive value is the highest.

\section{Transcranial MEPs}

In 1998, Kothbauer et al. ${ }^{8}$ presented their experience with 100 consecutive IMSCT resections. The specificity and sensitivity of combined data from epidural and muscle MEP recordings were $100 \%$ and $91 \%$, respectively, during the immediate postoperative period. Muscle MEP recordings heralded 40 cases with short-term neurological deterioration, leading to 5 false-positive alerts without any false-negative alerts. The authors reported a $100 \%$ neurological improvement rate, with all patients returning to their baseline status eventually, leading to the conclusion that MEP attenuations denote compromises to motor pathways before irreversible damage occurs. In 5 patients, the procedure was terminated due to intraoperative motor attenuations. In the majority of these patients (4 of 5), this led to an STR. More recently, Li et al. ${ }^{9}$ published their experience with 129 MEP recordings in the resection of IMSCTs and evaluated two postoperative time points: POD7 and 2 years postoperatively. At POD7, intraoperative MEP attenuations correctly heralded 28 cases of moderate to severe neurological deterioration. By 2 years postoperatively, 18 patients still had worse neurological function relative to their preoperative status. In another reported study, ${ }^{6}$ immediate postoperative neurological decline occurred in $34 \%$ of the patients undergoing intramedullary tumor removal. These authors reported a significant association between MEP attenuation and immediate resection-associated injury. However, the association was not consistent with the postoperative 1-month neurological status, in which $41 \%$ of the patients with deteriorated status had returned to their baseline status. Another study analyzed the sensitivity and specificity rates of IONM for 6-month outcomes in 14 patients with IMSCTs, and these authors found an unsatisfactory sensitivity rate of $37.5 \%$, albeit a specificity rate of $100 \% .^{14}$ This finding demonstrates the neuromonitoring limitations in predicting longterm outcomes. Recently, a meta-analysis of 21 studies $^{7}$ found MEP sensitivities in the range of 75\%-99\%, with a pooled sensitivity of $90 \%$ and a pooled specificity of $82 \%$ (range 27\%-97\%). The authors accurately noted the heterogeneity in the time of outcome measurements between the various studies as a limitation to their analysis. Our tcMEP data demonstrate relatively consistent sensitivities $(78 \%, 87 \%$, and $86 \%$ at POD1, PO-6 weeks, and last follow-up, respectively), albeit a fluctuating PPV which declined from 58\% at POD1 to 33\% in only 6 weeks. In our cohort, $25 \%$ of tcMEP attenuations accurately reflected the neurological status at the immediate postoperative period but have gradually lost relevance as the time from surgery elapsed.

\section{D-Wave Recording}

The placement of an epidural or subdural electrode in close proximity to the motor pathways allows for a more durable alternative than peripheral tcMEP recording and increases the resilience to anesthetic changes and to muscle relaxants. ${ }^{11,13,15}$ In one series, ${ }^{8} \mathrm{D}$-wave recording remained stable in all false-positive tcMEP changes. Costa et al. ${ }^{15}$ reported an NPV of $100 \%$ in their experience with 23 IMSCT operations. Importantly, the authors defined the neurological outcome as the last measured neurological status, rendering transient postoperative deficits that underwent complete spontaneous resolution as "neurologically intact." This definition differs from that used in the majority of publications in which the primary neurologi- 


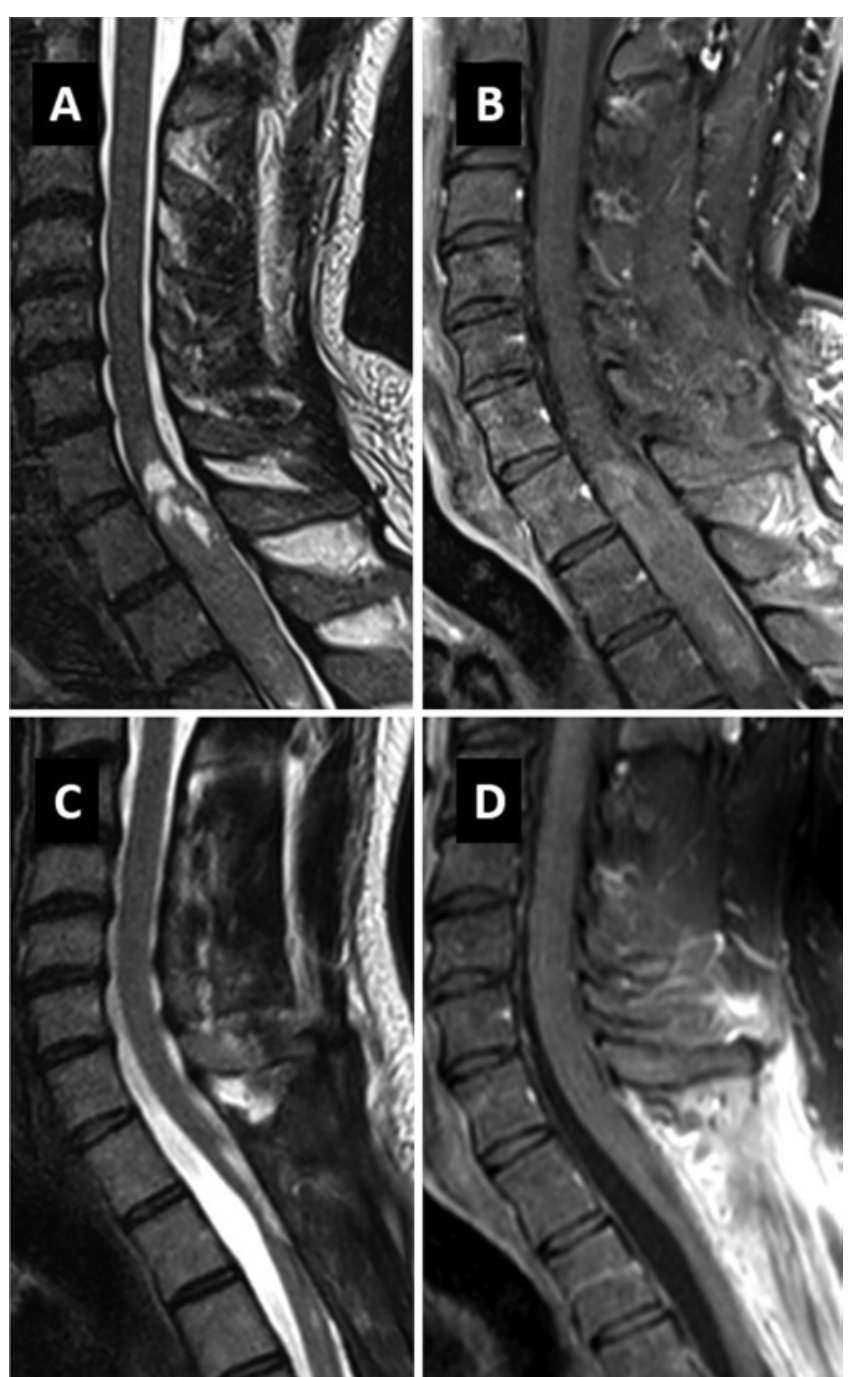

FIG. 2. MRI of intramedullary grade II ependymoma. A: T2-weighted midsagittal preoperative MR image. B: Post-gadolinium-injected T1-weighted preoperative midsagittal MR image. C: T2-weighted MR image obtained 21 months postoperatively demonstrating GTR of the tumor. D: Post-gadolinium-injected T1-weighted MR image obtained 21 months postoperatively without residual enhancing mass.

cal outcome was recorded during the immediate postoperative period. In our series, D-wave was obtained in 12 cases $(42.8 \%)$, as it is unattainable at the conus medullaris and above the $\mathrm{C} 2$ level. Among all modalities obtained, D-wave specificity was highest and the most consistent over time $(100 \%, 83 \%$, and $90 \%$ at POD1, PO-6 weeks, and last follow-up, respectively), surpassing the specificity of tcMEPs by more than twofold. The D-wave's NPV was higher at the long-term postoperative period, while its PPV declined by $50 \%$ between POD1 and PO- 6 weeks.

Striving to achieve GTR may be advisable in pathologies in which compete resection may be curative. In our cohort, GTR was feasible in the majority of patients $(60.7 \%$ overall). We found a significant association between GTR and ependymoma $(p=0.04)$, indicating the surgeons' preference to continue the resection despite a high rate of tcMEP and D-wave attenuations (77\%). Con- versely, astrocytoma was seldom completely resected (< $20 \%$ ) due to the relatively limited benefit of increasing the extent of resection on account of resection-associated injury.

In the face of persistent attenuation of motor potentials, the surgeon may opt to cease the resection or to continue nonetheless. Within the group in which the tumor removal was halted due to tcMEP or D-wave attenuations $(n=7)$, 3 patients were neurologically stable at POD1 relative to their baseline status (42.8\% among halted operations). The remaining 4 patients $(57.2 \%)$ had resection-associated injury at both the immediate and the long-term postoperative period. In comparison, within the group in which tumor resection was uninterrupted despite the neuromonitoring alert, 9 patients $(69 \%)$ had resection-associated injury at POD1, yet $85 \%$ of them returned to their baseline functional status by the time of their last follow-up. In 5 patients with immediate postoperative deterioration, Dwave recordings were uninterrupted. Four of these patients gradually improved to the same functional status as they were before surgery.

We believe that pursuing GTR in curable pathologies may be advisable even at the risk of severe temporary neurological deficits, but may be inappropriate at the cost of permanent neurological disability. This study exhibits a lower PPV of tcMEPs in the late postoperative period compared to the immediate postoperative period. This finding may indicate a limited role of tcMEPs in predicting long-term outcomes and should be considered in the surgical decision-making process.

\section{Somatosensory Evoked Potentials}

In a meta-analysis that evaluated the diagnostic utility of IONM, ${ }^{7}$ the overall sensitivity and specificity for SSEP monitoring ranged from $70 \%$ to $95 \%$ and from $61 \%$ to $96 \%$, respectively. Li et al. ${ }^{9}$ evaluated 155 SSEP recordings in ependymoma resections; in 79 operations, SSEP attenuation (51\%) was noted. Of these, only 28 patients (35\%) had a clinically significant sensory deterioration at 1 week from surgery, indicating a relatively low PPV of SSEP for the early postoperative period in that cohort. It should be noted, though, that the majority of these patients demonstrated a consistent sensory deficit throughout the longer postoperative period, with 24 out of 28 patients $(91 \%)$ maintaining their sensory deficit at 1 year postoperatively. Our results demonstrate low sensitivity rates throughout the follow-up period for SSEP, albeit a consistent specificity $(71.4,78.6$, and 70 at POD1, PO-6 weeks, and last follow-up, respectively). Our practice is to continue the tumor resection despite SSEP attenuations, as they may be the inherent result of the midline approach to dorsal column interruption at the beginning of surgery.

\section{Study Limitations}

The retrospective nature of this study renders it susceptible to the inherent weaknesses of all retrospective analyses. In particular, the crude nature of the McCormick scaling system may overlook minor postoperative deficits. In addition, the relatively small cohort limits the statistical power of this study. 


\section{Conclusions}

Even though the predictive value of IONM has been the focus of previous publications, inconsistencies in the time points in which the neurological outcome was measured may impede the applicability of these studies. The diagnostic accuracy of IONM in the resection of IMSCT varies throughout the postoperative period. The PPV of motor recordings decreased by $25 \%$ as the time from surgery elapsed, suggesting it is more predictive of short-term rather than long-term neurological deficits. The NPV of tcMEP has consistently increased, indicating a higher diagnostic accuracy in identifying patients who eventually preserve neurological function, albeit an increased proportion of false-negative results in the short-term perioperative period. Of note, D-wave recordings correctly heralded the majority of long-term neurological outcomes. This finding did not reach statistical significance due to the small sample size. The postoperative variations in the predictive value of IONM should be considered in the surgical decision-making process when weighing the risk of resection-associated neurological injury against the implications of partial tumor resection.

\section{References}

1. Samartzis D, Gillis CC, Shih P, et al. Intramedullary spinal cord tumors: Part II-management options and Outcomes. Global Spine J. 2016;6(2):176-185.

2. Tobin MK, Geraghty JR, Engelhard HH, et al. Intramedullary spinal cord tumors: a review of current and future treatment strategies. Neurosurg Focus. 2015;39(2):E14.

3. McCormick PC, Torres R, Post KD, Stein BM. Intramedullary ependymoma of the spinal cord. J Neurosurg. 1990; 72(4):523-532.

4. Samartzis D, Gillis CC, Shih P, et al. Intramedullary spinal cord tumors: Part I-epidemiology, pathophysiology, and diagnosis. Global Spine J. 2015;5(5):425-435.

5. Rashad S, Elwany A, Farhoud A. Surgery for spinal intramedullary tumors: technique, outcome and factors affecting resectability. Neurosurg Rev. 2018;41(2):503-511.

6. Garcés-Ambrossi GL, McGirt MJ, Mehta VA, et al. Factors associated with progression-free survival and long-term neurological outcome after resection of intramedullary spinal cord tumors: analysis of 101 consecutive cases. J Neurosurg Spine. 2009;11(5):591-599.

7. Azad TD, Pendharkar AV, Nguyen V, et al. Diagnostic utility of intraoperative neurophysiological monitoring for intramedullary spinal cord tumors: systematic review and metaanalysis. Clin Spine Surg. 2018;31(3):112-119.
8. Kothbauer KF, Deletis V, Epstein FJ. Motor-evoked potential monitoring for intramedullary spinal cord tumor surgery: correlation of clinical and neurophysiological data in a series of 100 consecutive procedures. Neurosurg Focus. 1998;4(5):e1.

9. Li TY, Chu JS, Xu YL, et al. Surgical strategies and outcomes of spinal ependymomas of different lengths: analysis of 210 patients: clinical article. J Neurosurg Spine. 2014; 21(2):249-259.

10. Kobayashi S, Matsuyama Y, Shinomiya K, et al. A new alarm point of transcranial electrical stimulation motor evoked potentials for intraoperative spinal cord monitoring: a prospective multicenter study from the Spinal Cord Monitoring Working Group of the Japanese Society for Spine Surgery and Related Research. J Neurosurg Spine. 2014;20(1):102107.

11. Morota N, Deletis V, Constantini S, et al. The role of motor evoked potentials during surgery for intramedullary spinal cord tumors. Neurosurgery. 1997;41(6):1327-1336.

12. Sala F, Palandri G, Basso E, et al. Motor evoked potential monitoring improves outcome after surgery for intramedullary spinal cord tumors: a historical control study. Neurosurgery. 2006;58(6):1129-1143.

13. Sala F, Bricolo A, Faccioli F, et al. Surgery for intramedullary spinal cord tumors: the role of intraoperative (neurophysiological) monitoring. Eur Spine J. 2007;16(suppl 2): S130-S139.

14. Lakomkin N, Mistry AM, Zuckerman SL, et al. Utility of intraoperative monitoring in the resection of spinal cord tumors: an analysis by tumor location and anatomical region. Spine (Phila Pa 1976). 2018;43(4):287-294.

15. Costa P, Peretta P, Faccani G. Relevance of intraoperative D wave in spine and spinal cord surgeries. Eur Spine J. 2013; 22(4):840-848.

\section{Disclosures}

The authors report no conflict of interest concerning the materials or methods used in this study or the findings specified in this paper.

\section{Author Contributions}

Conception and design: Harel, Kimchi, Knoller. Acquisition of data: Kimchi, Eyal-Mazuz, Peled. Analysis and interpretation of data: Harel, Kimchi. Drafting the article: Harel, Kimchi,

Knoller. Critically revising the article: Harel, Knoller, Korn, Sapir. Reviewed submitted version of manuscript: all authors. Approved the final version of the manuscript on behalf of all authors: Harel. Statistical analysis: Kimchi. Study supervision: Harel.

\section{Correspondence}

Ran Harel: Sheba Medical Center, Ramat-Gan, Israel. ran.harel@ sheba.health.gov.il. 